PROCEEDINGS OF THE

AMERICAN MATHEMATICAL SOCIETY

Volume 139, Number 3, March 2011, Pages 897-907

S 0002-9939(2010)10477-X

Article electronically published on October 28, 2010

\title{
NON-HAUSDORFF ÉTALE GROUPOIDS
}

\author{
R. EXEL
}

(Communicated by Marius Junge)

\begin{abstract}
We present examples of non-Hausdorff, étale, essentially principal groupoids for which three results, known to hold in the Hausdorff case, fail. These results are: (A) the subalgebra of continuous functions on the unit space is maximal abelian within the reduced groupoid $\mathrm{C}^{*}$-algebra, $(\mathrm{B})$ every nonzero ideal of the reduced groupoid $\mathrm{C}^{*}$-algebra has a nonzero intersection with the subalgebra of continuous functions on the unit space, and $(\mathrm{C})$ the open support of a normalizer is a bisection.
\end{abstract}

\section{INTRODUCTION}

This paper is concerned with étale groupoids 10, 1, 9, 11, 4. A topological groupoid $G$ is said to be étale if its unit space $G^{(0)}$ is locally compact and Hausdorff, and if the range map " $r$ " (and consequently also the source map " $s$ ") is a local homeomorphism.

One may or may not assume the global topology of $G$ to be Hausdorff, but, while non-Hausdorff topological spaces may be safely ignored in numerous applications of Topology, non-Hausdorff groupoids do occur in many essential situations, such as the holonomy groupoid of a foliation [2] or the groupoid of germs of a pseudogroup of local homeomorphisms on a topological space [11, Section 3].

Therefore, rather than dismissing non-Hausdorff groupoids as a nuisance, it is highly desirable to embrace them in the general theory.

An étale groupoid $G$ is said to be principal if its isotropy group bundle, namely

$$
G^{\prime}:=\{\gamma \in G: s(\gamma)=r(\gamma)\},
$$

coincides with the unit space $G^{(0)}$, and it is said to be essentially principal if the interior of $G^{\prime}$ coincides with $G^{(0)}$. Principal groupoids correspond to free group actions, while the essentially principal ones correspond to topologically free actions, hence the relevance of these concepts.

Among the important consequences of the property of being essentially principal in the Hausdorff case, the following stand out:

(A) $C_{0}\left(G^{(0)}\right)$ is maximal abelian within the reduced groupoid $\mathrm{C}^{*}$-algebra $C_{r}^{*}(G)$ [11, 4.2].

(B) Every nonzero ideal of $C_{r}^{*}(G)$ has a nonzero intersection with $C_{0}\left(G^{(0)}\right)$ (see the appendix for a precise statement).

Received by the editors July 30, 2009.

2010 Mathematics Subject Classification. Primary 46L55; Secondary 22A22.

Key words and phrases. Non-Hausdorff groupoids, essentially principal groupoids.

The author was partially supported by CNPq. 
(C) If $u$ is a normalizer of $C_{0}\left(G^{(0)}\right)$ within $C_{r}^{*}(G)$, then the open support of $u$, namely

$$
\operatorname{supp}^{\prime}(u)=\{\gamma \in G: u(\gamma) \neq 0\},
$$

is a bisection [11, Proposition 4.7].

These results underlie mainstream developments in the theory of $\mathrm{C}^{*}$-algebras: (B) is related to uniqueness theorems for Cuntz-Krieger algebras [3, 2.15], 5, 13.2] and graph algebras [8], as well as to results on reduced crossed products by partial group actions [6, 2.6], while (A) and (C) are related to Cartan subalgebras [7, 11.

It should be stressed that (A), (B) and (C) are only known to hold under the assumption that $G$ is Hausdorff!

In trying to embrace non-Hausdorff groupoids within the general theory, the author (and quite likely many other people) has spent a lot of energy in an effort to generalize the above facts beyond the Hausdorff case. After having failed to do so he has found examples of non-Hausdorff étale groupoids which provide counterexamples for all of the above statements. In what follows we shall discuss these examples in detail.

Our first example is related to an example by G. Skandalis [12 built with a different purpose, namely that of exhibiting a minimal foliation whose $\mathrm{C}^{*}$-algebra is not simple.

The author would like to thank Jean Renault for many fruitful discussions and for bringing Skandalis' example to his attention. He would also like to thank Alcides Buss for many interesting discussions while the author was searching for the second example below.

\section{THE FIRST EXAMPLE}

Consider the following subsets of $\mathbb{R}^{2}$ :

$$
\begin{aligned}
& X=[-1,1] \times\{0\}, \\
& Y=\{0\} \times[-1,1], \\
& Z=X \cup Y .
\end{aligned}
$$

Clearly $Z$ is invariant under the action of the subgroup $H \subseteq G L_{2}(\mathbb{R})$ generated by

$$
\sigma_{x}=\left(\begin{array}{cc}
-1 & 0 \\
0 & 1
\end{array}\right) \quad \text { and } \quad \sigma_{y}=\left(\begin{array}{cc}
1 & 0 \\
0 & -1
\end{array}\right)
$$

Let $G$ be the groupoid of germs for the action of $H$ on $Z$ (see Section (3) of [1] for the definition of the groupoid of germs for a given pseudogroup). As is the case for every groupoid of germs, $G$ is essentially principal [11, 3.4].

We shall adopt a slightly simplified notation in relation to [11, namely the germ of the transformation $\varphi$ at the point $x$ will be denoted by $[\varphi, x]$, as opposed to Renault's notation $[y, \varphi, x]$, where $y=\varphi(x)$.

In the present case it is interesting to observe that

$$
\begin{array}{ll}
{\left[\sigma_{y}, x\right]=[I, x],} & {\left[\sigma_{x} \sigma_{y}, x\right]=\left[\sigma_{x}, x\right],} \\
{\left[\sigma_{x}, y\right]=[I, y],} & {\left[\sigma_{x} \sigma_{y}, y\right]=\left[\sigma_{y}, y\right],}
\end{array}
$$

for all $x \in X^{*}:=X \backslash\{0\}$, and all $y \in Y^{*}:=Y \backslash\{0\}$, where " $I$ " stands for the identity map and where we denote the zero vector of $\mathbb{R}^{2}$ simply by " 0 ". We therefore see 
that $G$ consists of the following distinct elements:

- $\quad[I, x]$, for $x \in X^{*}$,

- $\left[\sigma_{x}, x\right]$, for $x \in X^{*}$,

- $[I, 0]$,

- $\left[\sigma_{y}, 0\right]$,
- $[I, y]$, for $y \in Y^{*}$,

- $\left[\sigma_{y}, y\right]$, for $y \in Y^{*}$,

- $\left[\sigma_{x}, 0\right]$,

- $\left[\sigma_{x} \sigma_{y}, 0\right]$.

Observe that the isotropy group bundle $G^{\prime}$ is formed by the last three elements listed above, in addition to the units.

Recall that a bisection is a subset of $G$ restricted to which both the range and source maps are injective. Consider the following open bisections of $G$ :

- $U_{1}=\{[I, z]: z \in Z\}=G^{(0)}$,

- $U_{y}=\left\{\left[\sigma_{y}, z\right]: z \in Z\right\}$,
- $U_{x}=\left\{\left[\sigma_{x}, z\right]: z \in Z\right\}$,

- $U_{x y}=\left\{\left[\sigma_{x} \sigma_{y}, z\right]: z \in Z\right\}$.

Let $f_{1}, f_{x}, f_{y}, f_{x y} \in C_{c}(G)$ (for the definition of $C_{c}(G)$ see [2], 9] or [4, 3.9]) be the characteristic function of $U_{1}, U_{x}, U_{y}$, and $U_{x y}$, respectively. Finally put

$$
f=f_{1}-f_{x}-f_{y}+f_{x y} .
$$

By direct computation one checks that

$$
\begin{array}{ll}
\text { - } f([I, 0])=1, & \bullet f\left(\left[\sigma_{x}, 0\right]\right)=-1, \\
\text { - } f\left(\left[\sigma_{y}, 0\right]\right)=-1, & \bullet f\left(\left[\sigma_{x} \sigma_{y}, 0\right]\right)=1
\end{array}
$$

and that $f$ vanishes on all other points of $G$. In particular notice that the support of $f$ (the set of points where $f$ does not vanish, no closure) is the set

$$
\left\{[I, 0],\left[\sigma_{x}, 0\right],\left[\sigma_{y}, 0\right],\left[\sigma_{x} \sigma_{y}, 0\right]\right\}=r^{-1}(\{0\})=s^{-1}(\{0\}),
$$

which is contained in $G^{\prime}$.

2.1. Proposition. For every $g \in C_{c}(G)$ one has that

$$
g * f=f * g=\lambda(g) f
$$

where $\lambda(g)$ is the scalar given by

$$
\lambda(g)=g([I, 0])-g\left(\left[\sigma_{x}, 0\right]\right)-g\left(\left[\sigma_{y}, 0\right]\right)+g\left(\left[\sigma_{x} \sigma_{y}, 0\right]\right) .
$$

Proof. Recall that for every $\gamma \in G$ one has

$$
(f * g)(\gamma)=\sum_{\alpha \beta=\gamma} f(\alpha) g(\beta), \quad \forall \gamma \in G .
$$

If the above sum is nonzero, then there exists at least one pair $(\alpha, \beta)$ such that $\alpha \beta=\gamma$ and $f(\alpha) \neq 0$. As seen in (2.1), this implies that $r(\alpha)=0$, and hence necessarily $r(\gamma)=0$, as well. Therefore $f * g$ is supported in $r^{-1}(\{0\})$. A similar reasoning and the same conclusion apply to $g * f$.

We leave it to the reader to compute $(f * g)(\gamma)$ and $(g * f)(\gamma)$ for the four elements $\gamma$ in $r^{-1}(\{0\})$, after which the result will become apparent.

The first conclusion to be drawn from the above result is:

2.2. Proposition. Even though $G$ is essentially principal (every groupoid of germs is essentially principal by [11,3.4]), there is a nonzero ideal $J \subseteq C_{r}^{*}(G)$ for which $J \cap C_{0}\left(G^{(0)}\right)=\{0\}$.

Proof. By Proposition 2.2 one has that $J:=\mathbb{C} f$ is an ideal in $C_{r}^{*}(G)$. Since $f$ is not in $C_{0}\left(G^{(0)}\right)$, the intersection of $J$ with $C_{0}\left(G^{(0)}\right)$ is trivial. 
The second conclusion is:

2.3. Proposition. Even though $G$ is essentially principal, one has that $C_{0}\left(G^{(0)}\right)$ is not maximal abelian within $C_{r}^{*}(G)$.

Proof. It is enough to notice that by Proposition 2.2 one has that $f$ is a central element of $C_{r}^{*}(G)$, and hence commutes with every element of $C_{0}\left(G^{(0)}\right)$, but $f$ is not in $C_{0}\left(G^{(0)}\right)$.

Since the support of $f$ is contained in $G^{\prime}$, and in view of [11,4.2], it is not surprising that $f$ commutes with every element of $C_{0}\left(G^{(0)}\right)$.

\section{Strange NORMALIZERS}

Let $n$ be a positive integer and let $I=I_{n}=\{1,2, \ldots, n\}$ be seen as a discrete topological space. On the product space $[0,1] \times I$, we consider the equivalence relation " " according to which

$$
(0, i) \sim(0, j), \quad \forall i, j \in I,
$$

and such that no other pairs of points are related except for each point with itself. The quotient topological space

$$
X=([0,1] \times I) / \sim
$$

therefore looks like a star with $n$ edges. Incidentally, in the special and very relevant case $n=4$, notice that $X$ is homeomorphic to the space $Z$ of the previous section.

The equivalence class of $(0, i)$ will be denoted simply by 0 , and if $t>0$, the equivalence class of $(t, i)$, namely the singleton $\{(t, i)\}$, will be denoted by $(t, i)$ by abuse of language.

Let $S_{n}$ be the group of permutations of $I$ and consider the action of $S_{n}$ on $[0,1] \times I$, where each $\sigma \in S_{n}$ acts by

$$
(t, i) \mapsto(t, \sigma(i))
$$

The equivalence relation " $\sim$ " above is clearly left invariant by this action, so we get an action of $S_{n}$ on $X$. Considering the subgroup $A_{n} \subseteq S_{n}$ formed by all even permutations, we may restrict the above action to $A_{n}$, and we shall let

$$
G=G\left(X, A_{n}\right)
$$

be the corresponding groupoid of germs. The unit space of $G$ is therefore homeomorphic to $X$, and we shall tacitly identify these from now on.

Notice that if $x=(t, i)$, with $t>0$, and if $\sigma, \sigma^{\prime} \in A_{n}$, then

$$
[\sigma,(t, i)]=\left[\sigma^{\prime},(t, i)\right] \Longleftrightarrow \sigma(i)=\sigma^{\prime}(i) .
$$

In other words, the germ of $\sigma$ at $(t, i)$ depends only on $j:=\sigma(i)$. We may therefore denote this germ simply by $(t, i, j)$. On the other hand, it is easy to see that

$$
[\sigma, 0]=\left[\sigma^{\prime}, 0\right] \Longleftrightarrow \sigma=\sigma^{\prime} .
$$

We may then describe $G$ as being the set

$$
\{(t, i, j): t \in(0,1], i, j \in I\} \quad \cup \quad\left\{[\sigma, 0]: \sigma \in A_{n}\right\} .
$$

The main technical result of this section is in order: 
3.1. Theorem. Assuming that $n \geq 4$, and given any $\tau \in S_{n}$ (not necessarily in $\left.A_{n}\right)$, there exists a unitary element $u$ in $C_{r}^{*}(G)$ such that for all $t>0$ and all $i, j \in I$, one has

$$
u(t, i, j)= \begin{cases}1, & \text { if } \tau(i)=j, \\ 0, & \text { otherwise. }\end{cases}
$$

Moreover, $u^{*} f u=f \circ \tau$ for every $f \in C_{0}(X)$.

Proof. For each $\sigma \in A_{n}$, let $U_{\sigma}=\{[\sigma, x]: x \in X\}$ be the canonical bisection associated to $\sigma$. Since $U_{\sigma}$ is compact, one has that its characteristic function, here denoted $1_{\sigma}$, is an element of $C_{r}^{*}(G)$, which is easily seen to be unitary. Moreover, the correspondence

$$
\sigma \in A_{n} \mapsto 1_{\sigma} \in C_{r}^{*}(G)
$$

is a unitary representation of $A_{n}$ in $C_{r}^{*}(G)$, which therefore integrates to a *-homomorphism

Given a generic element

$$
\phi: C^{*}\left(A_{n}\right) \rightarrow C_{r}^{*}(G)
$$

$$
a=\sum_{\sigma \in A_{n}} a_{\sigma} \delta_{\sigma} \in C^{*}\left(A_{n}\right)
$$

and any $(t, i, j) \in G$, with $t>0$, observe that

$$
\phi(a)(t, i, j)=\sum_{\sigma \in A_{n}} a_{\sigma} 1_{\sigma}(t, i, j)=\sum_{\sigma(i)=j} a_{\sigma} .
$$

Changing subjects slightly, consider the representation $\pi$ of $S_{n}$ on the Hilbert space $\mathbb{C}^{n}$, where each $\sigma \in S_{n}$ is mapped to the unitary operator $\pi(\sigma)$ defined on the cannonical basis $\left\{e_{i}\right\}_{i \in I}$ of $\mathbb{C}^{n}$ by

$$
\pi(\sigma) e_{i}=e_{\sigma(i)} .
$$

Denote by $\tilde{\pi}$ the corresponding integrated representation of $C^{*}\left(S_{n}\right)$ on $\mathbb{C}^{n}$, and observe that for each $a$ as in (3.1.1) one has that

$$
\left\langle\tilde{\pi}(a) e_{i}, e_{j}\right\rangle=\sum_{\sigma \in A_{n}} a_{\sigma}\left\langle\pi(\sigma) e_{i}, e_{j}\right\rangle=\sum_{\sigma(i)=j} a_{\sigma},
$$

so we have by (3.1.2) that

$$
\phi(a)(t, i, j)=\left\langle\tilde{\pi}(a) e_{i}, e_{j}\right\rangle,
$$

for all $a \in C^{*}\left(A_{n}\right)$, all $t \in(0,1]$, and all $i, j \in I$.

Assuming that $n \geq 4$, one may prove that the commutant in $\mathcal{B}\left(\mathbb{C}^{n}\right)$ of both $\tilde{\pi}\left(C^{*}\left(S_{n}\right)\right)$ and $\tilde{\pi}\left(C^{*}\left(A_{n}\right)\right)$ coincide with the set of all matrices of the form

$$
\left(\begin{array}{ccccc}
z & y & y & \ldots & y \\
y & z & y & \ldots & y \\
y & y & z & \ldots & y \\
\vdots & \vdots & \vdots & \ddots & \vdots \\
y & y & y & \ldots & z
\end{array}\right),
$$

where $z, y \in \mathbb{C}$. The crucial point in doing this is that $A_{n}$ acts bi-transitively on $I$, meaning that given $i_{1} \neq i_{2}$ and $j_{1} \neq j_{2}$, there exists $\sigma \in A_{n}$ such that $\sigma\left(i_{1}\right)=j_{1}$ and $\sigma\left(i_{2}\right)=j_{2}$. Incidentally, this is not true for $n<4$. 
By the double commutant theorem we conclude that $\tilde{\pi}\left(C^{*}\left(S_{n}\right)\right)=\tilde{\pi}\left(C^{*}\left(A_{n}\right)\right)$. Given $\tau \in S_{n}$, as in the theorem, we therefore have that $\pi(\tau) \in \tilde{\pi}\left(C^{*}\left(A_{n}\right)\right)$, so there exists some $v \in C^{*}\left(A_{n}\right)$ such that $\tilde{\pi}(v)=\pi(\tau)$. Absent any K-theoretic obstructions, we may assume that $v$ is unitary.

The element $u$ of which the theorem speaks is the unitary element $u:=\phi(v) \in$ $C_{r}^{*}(G)$. To see that it satisfies the required conditions, notice that for all $t>0$ and $i, j \in I$ we have that

$$
u(t, i, j)=\phi(v)(t, i, j)=\left\langle\tilde{\pi}(v) e_{i}, e_{j}\right\rangle=\left\langle\pi(\tau) e_{i}, e_{j}\right\rangle=\left\langle e_{\tau(i)}, e_{j}\right\rangle,
$$

proving the first assertion. It follows that the open support of $u$, namely

$$
\operatorname{supp}^{\prime}(u)=\{\gamma \in G: u(\gamma) \neq 0\}
$$

(cf. [11]), consists precisely of all germs $(t, i, \tau(i))$, where $t>0$, besides a few other germs at 0 . Therefore the range of any $\gamma \in \operatorname{supp}^{\prime}(u)$ coincides whith the image of its source under the action of $\tau$. From this it immediately follows that

$$
u^{*} f u=f \circ \tau, \quad \forall f \in C_{0}(X) .
$$

The relevance of this result is in relation to [11, Proposition 4.7], where it is proved that the open support of a normalizer of $C_{0}(X)$ in $C_{r}^{*}(G)$ is a bisection. In the present non-Hausdorff situation this fails:

3.1. Proposition. Let $n \geq 4$, let $\tau \in S_{n} \backslash A_{n}$, and let $u$ be given as in Theorem 3.1 . Then $u$ is a normalizer of $C_{0}\left(G^{(0)}\right)$ within $C_{r}^{*}(G)$, but even though $G$ is essentially principal, the open support of $u$ is not a bisection.

Proof. Recall from the proof of Theorem 3.1 that $u=\phi(v)$, where $v$ is a unitary element in $C^{*}\left(A_{n}\right)$ such that $\tilde{\pi}(v)=\pi(\tau)$. Assuming by contradiction that $\operatorname{supp}^{\prime}(u)$ is a bisection and noticing that the germs $[\sigma, 0]$ all have range and source equal to 0 , we deduce that there is at most one $\sigma \in A_{n}$ for which $u([\sigma, 0]) \neq 0$.

Write $v=\sum_{\sigma \in A_{n}} a_{\sigma} \delta_{\sigma}$, as in (3.1.1), so that $u=\sum_{\sigma \in A_{n}} a_{\sigma} 1_{\sigma}$, and hence

$$
u([\sigma, 0])=a_{\sigma}, \quad \forall \sigma \in A_{n} .
$$

We then see that there is only one $\sigma \in A_{n}$ for which $a_{\sigma} \neq 0$, which implies that $v=a_{\sigma} \delta_{\sigma}$. Consequently

$$
\pi(\tau)=\tilde{\pi}(v)=a_{\sigma} \pi(\sigma),
$$

which contradicts the fact that $\tau \notin A_{n}$.

Another dilemma presented by this example is related to the program initiated by Kumjian in [7], and recently continued by Renault in [11, attempting to classify Cartan sub-algebras of $\mathrm{C}^{*}$-algebras. Given a commutative subalgebra $B$ of a $\mathrm{C}^{*}$ algebra $A$ and a normalizer $u \in N(B)$, Kumjian [7] showed the existence of a partial homeomorphism $\theta_{u}$ of the spectrum $X$ of $B$, such that for all $x \in X$, with $\left(u^{*} u\right)(x) \neq 0$, one has

$$
\left(u^{*} b u\right)(x)=\left(u^{*} u\right)(x) b\left(\theta_{u}(x)\right), \quad \forall b \in B .
$$

When $A$ is the reduced $\mathrm{C}^{*}$-algebra of an essentially principal, Hausdorff, étale groupoid $G$, and $B=C_{0}\left(G^{(0)}\right)$, Renault showed 11] that one can reconstruct $G$ from the inclusion " $B \subseteq A$ ", as the germs of the partial homeomorphisms $\theta_{u}$, where $u$ ranges in the set of all normalizers.

In the present example, if one attempted to reconstruct $G$ from the inclusion " $C_{0}(X) \subseteq C_{r}^{*}(G)$ " using the above method, the presence of the strange normalizers 
$u$ above would lead us to consider the germ at zero of every $\tau \in S_{n}$ by the last assertion of Theorem 3.1, but the isotropy group $G(0)$ is only as large as $A_{n}$ !

\section{ApPENDix (The INTERSECTION PROPERTY FOR IDEALS IN ESSENTIALLY PRINCIPAL, HAUSDORFF GROUPOIDS)}

In this section we prove result (B) stated in the introduction. Although this result has been used in several contexts under various guises (see the introduction for some references), it seems not to have appeared in the literature in quite the general form we have in mind. We begin with some elementary considerations about representations of commutative $\mathrm{C}^{*}$-algebras.

Let $X$ be a locally compact Hausdorff space and let $\pi$ be a representation of $C_{0}(X)$ on a Hilbert space $H$. As any ideal of $C_{0}(X)$, the kernel of $\pi$ must be of the form $C_{0}(U)$, for some open set $U \subseteq X$.

4.1. Definition. Given a representation $\pi$ of $C_{0}(X)$, with $\operatorname{Ker}(\pi)=C_{0}(U)$, we will refer to $X \backslash U$ as the support of $\pi$.

4.2. Lemma. If $\pi$ is a representation of $C_{0}(X)$ on a Hilbert space $H$, and if $x$ lies in the support of $\pi$, then $|f(x)| \leq\|\pi(f)\|$, for every $f$ in $C_{0}(X)$.

Proof. This is left to the reader.

Given a groupoid $G$, for every $x \in G^{(0)}$ we denote by $G(x)$ (cf. [10, I.1.1]) the isotropy group at $x$, namely

$$
G(x)=\{\gamma \in G: s(\gamma)=r(\gamma)=x\} .
$$

Obviously $x \in G(x)$, but in case $G(x)=\{x\}$ we say that $x$ has no isotropy.

The following result gives the key inequality from which the next theorem will be deduced. It is roughly based on [10, II.4.4].

4.3. Lemma. Let $G$ be an étale, Hausdorff groupoid and let $\pi$ be a representation of $C^{*}(G)$ on a Hilbert space $H$. Suppose in addition that we are given $x \in G^{(0)}$ such that

(i) $x$ has no isotropy,

(ii) $x$ lies in the support of $\left.\pi\right|_{C_{0}\left(G^{(0)}\right)}$.

Then for every $f \in C_{c}(G)$, one has that $|f(x)| \leq\|\pi(f)\|$.

Proof. Let $\mathcal{V}$ be the collection of all open neighborhoods of $x$ within $G^{(0)}$. We will view $\mathcal{V}$ as a directed set under the order relation

$$
v \leq w \Longleftrightarrow v \supseteq w, \quad \forall v, w \in \mathcal{V} .
$$

For each $v \in \mathcal{V}$, choose $v_{1}, v_{2} \in \mathcal{V}$, relatively compact, and such that $\bar{v}_{2} \subseteq v_{1} \subseteq$ $\bar{v}_{1} \subseteq v$. By Uryshon's Lemma let

$$
g_{v}: G^{(0)} \rightarrow[0,1]
$$

be a continuous function whose restriction to $\bar{v}_{2}$ is identically equal to 1 and which vanishes off $v_{1}$. The support of $g_{v}$ is contained in $\bar{v}_{1}$, which is compact, so $g_{v} \in$ $C_{c}\left(G^{(0)}\right)$.

We claim that there exists $\xi_{v} \in H$, with $\left\|\xi_{v}\right\|=1$, and such that

$$
\pi\left(g_{v}\right) \xi_{v}=\xi_{v} \text {. }
$$


In order to prove it, use Uryshon's Lemma again to produce a continuous function $h_{v}: G^{(0)} \rightarrow[0,1]$, vanishing off $v_{2}$ and such that $h_{v}(x) \neq 0$. Observe that, since $g_{v}$ is identically equal to 1 on $v_{2}$, we have that

$$
g_{v} h_{v}=h_{v} .
$$

From (ii) and Lemma 4.2 it follows that

$$
0<\left|h_{v}(x)\right| \leq\left\|\pi\left(h_{v}\right)\right\|,
$$

so $\pi\left(h_{v}\right) \neq 0$, and one may pick $\eta_{v} \in H$ such that $\left\|\pi\left(h_{v}\right) \eta_{v}\right\|=1$. Setting $\xi_{v}=$ $\pi\left(h_{v}\right) \eta_{v}$, we have that

$$
\pi\left(g_{v}\right) \xi_{v}=\pi\left(g_{v}\right) \pi\left(h_{v}\right) \eta_{v}=\pi\left(g_{v} h_{v}\right) \eta_{v} \stackrel{[4.3 .1]}{-} \pi\left(h_{v}\right) \eta_{v}=\xi_{v},
$$

proving our claim.

We next claim that

$$
\lim _{v \in \mathcal{V}}\left\langle\pi(f) \xi_{v}, \xi_{v}\right\rangle=f(x), \quad \forall f \in C_{c}(G) .
$$

Without loss of generality we will suppose that there are $K, U \subseteq G$ such that $K$ is compact, $U$ is an open bisection, $K \subseteq U$, and $f$ vanishes outside of $K$. We will further denote by $\alpha_{U}$ the homeomorphism from $s(U)$ to $r(U)$ given by

$$
\alpha_{U}(s(\gamma))=r(\gamma), \quad \forall \gamma \in U .
$$

The proof of (4.3.2) will be broken up in the following three cases:

(a) $x \notin s(U)$,

(b) $x \in s(U)$, and $\alpha_{U}(x) \neq x$,

(c) $x \in s(U)$, and $\alpha_{U}(x)=x$.

Proof of (4.3.2) under (a). Noticing that $x \notin s(K)$, there exists some $v_{0} \in \mathcal{V}$, with $v_{0} \cap s(K)=\emptyset$. For every $v \subseteq v_{0}$, one then has that

$$
\left(f g_{v}\right)(\gamma)=f(\gamma) g_{v}(s(\gamma))=0, \quad \forall \gamma \in G,
$$

because either $\gamma \notin K$ or $s(\gamma) \in s(K)$. Therefore $\pi(f) \xi_{v}=\pi\left(f g_{v}\right) \xi_{v}=0$, proving that the left-hand side of (4.3.2) vanishes. Observing that $x \notin K$ (or else $x=$ $s(x) \in s(K) \subseteq s(U))$, we see that the right-hand side of (4.3.2) also vanishes.

Proof of (4.3.2) under (b). Let $A$ and $B$ be pairwise disjoint open subsets of $G^{(0)}$ such that $x \in A$ and $\alpha_{U}(x) \in B$. Setting $v_{0}=A \cap \alpha_{U}^{-1}(B)$, notice that $x \in v_{0}$ and that $v_{0} \cap \alpha_{U}\left(v_{0}\right)=\emptyset$. For every $v \subseteq v_{0}$ we have that

$$
\left(g_{v}^{*} f g_{v}\right)(\gamma)=\overline{g_{v}(r(\gamma))} f(\gamma) g_{v}(s(\gamma)), \quad \forall \gamma \in G .
$$

If the above is nonzero for some $\gamma$, then $\gamma \in U$ and both $s(\gamma)$ and $r(\gamma)$ lie in $v$. Therefore

$$
r(\gamma)=\alpha_{U}(s(\gamma)) \in v \cap \alpha_{U}(v) \subseteq v_{0} \cap \alpha_{U}\left(v_{0}\right)=\emptyset,
$$

which is impossible. So $g_{v}^{*} f g_{v}=0$, and hence

$$
\left\langle\pi(f) \xi_{v}, \xi_{v}\right\rangle=\left\langle\pi(f) \pi\left(g_{v}\right) \xi_{v}, \pi\left(g_{v}\right) \xi_{v}\right\rangle=\left\langle\pi\left(g_{v}^{*} f g_{v}\right) \xi_{v}, \xi_{v}\right\rangle=0,
$$

again proving that the left-hand side of (4.3.2) vanishes. As for the right-hand side, notice that $x \notin U$, because otherwise

$$
\alpha_{U}(x)=\alpha_{U}(s(x))=r(x)=x,
$$

which is not in accordance with (b). Thus $f(x)=0$, and (4.3.2) is verified in the present case. 
Proof of (4.3.2) under (c). Given that $\alpha_{U}(x)=x$, there exists $\gamma \in U$ such that $s(\gamma)=r(\gamma)=x$. Thus

$$
\gamma \in G(x)=\{x\}
$$

so $\gamma=x$, by (i), and hence $x \in U$. As $G$ is assumed to be Hausdorff, we have that $f$ is continuous 1 so, given $\varepsilon>0$, we may choose a neighborhood $v_{0}$ of $x$, contained in $U \cap G^{(0)}$ and such that

$$
y \in v_{0} \Rightarrow|f(x)-f(y)| \leq \varepsilon .
$$

For every $v \subseteq v_{0}$ we have that

$$
\left(f g_{v}\right)(\gamma)=f(\gamma) g_{v}(s(\gamma)), \quad \forall \gamma \in G
$$

If the above is nonzero for some $\gamma$, then $\gamma \in U$ and $s(\gamma) \in v$. Thus, both $\gamma$ and $s(\gamma)$ lie in $U$, and since these have the same source, we deduce that $\gamma=s(\gamma)$, and hence that $\gamma \in v$. It follows that $f g_{v}$ vanishes outside $v$ and, in particular, $f g_{v} \in C_{0}\left(G^{(0)}\right)$. On the other hand, for every $y \in v$, one has that

$$
\left|\left(f g_{v}\right)(y)-f(x) g_{v}(y)\right|=|f(y)-f(x)|\left|g_{v}(y)\right| \leq \varepsilon\left\|g_{v}\right\|=\varepsilon,
$$

which gives $\left\|f g_{v}-f(x) g_{v}\right\| \leq \varepsilon$. Therefore, for $v$ as above,

$$
\begin{aligned}
& \left|\left\langle\pi(f) \xi_{v}, \xi_{v}\right\rangle-f(x)\right|=\left|\left\langle\pi(f) \xi_{v}, \xi_{v}\right\rangle-\left\langle f(x) \xi_{v}, \xi_{v}\right\rangle\right| \\
& \quad=\left|\left\langle\pi\left(f g_{v}\right) \xi_{v}, \xi_{v}\right\rangle-\left\langle f(x) \pi\left(g_{v}\right) \xi_{v}, \xi_{v}\right\rangle\right| \\
& \quad=\left|\left\langle\pi\left(f g_{v}-f(x) g_{v}\right) \xi_{v}, \xi_{v}\right\rangle\right| \leq\left\|f g_{v}-f(x) g_{v}\right\|\left\|\xi_{v}\right\|\left\|\xi_{v}\right\| \leq \varepsilon,
\end{aligned}
$$

proving (4.3.2) under the last case. We then finally get

$$
|f(x)|^{\stackrel{(4.3 .2)}{-}} \lim _{v \in \mathcal{V}}\left|\left\langle\pi(f) \xi_{v}, \xi_{v}\right\rangle\right| \leq\|\pi(f)\| .
$$

We are now ready to prove the precise form of result (B) stated in the introduction.

4.4. Theorem. Let $G$ be an étale, Hausdorff, essentially principal, second countable groupoid.

(a) If $\pi$ is a representation of $C_{r}^{*}(G)$ such that $\pi$ is faithful on $C_{0}\left(G^{(0)}\right)$, then $\pi$ is faithful.

(b) If $J$ is a nonzero ideal in $C_{r}^{*}(G)$, then $J \cap C_{0}\left(G^{(0)}\right)$ is nonzero.

Proof. We address (a) first. Since $\pi$ is assumed to be faithful on $C_{0}\left(G^{(0)}\right)$, the support of $\left.\pi\right|_{C_{0}\left(G^{(0)}\right)}$ is the whole of $G^{(0)}$. Given $f \in C_{c}(G)$ one then has by Lemma 4.3 that

$$
|f(x)| \leq\|\pi(f)\|,
$$

for every $x$ in $G^{(0)}$ without entropy. Employing [11, 3.1] we see that the set of such $x$ 's is dense in $G^{(0)}$, and since the restriction of $f$ to $G^{(0)}$ is continuous 2 we conclude that in fact (4.4.1) holds for every $x \in G^{(0)}$, so

$$
\sup _{x \in G^{(0)}}|f(x)| \leq\|\pi(f)\| \text {. }
$$

\footnotetext{
${ }^{1}$ On a non-Hausdorff groupoid the accepted definition of $C_{c}(G)$ (see [2, 9] or [4 3.9]) includes functions which are discontinuous, so we truly need to assume $G$ to be Hausdorff here.

${ }^{2}$ Here we again need to assume that $G$ is Hausdorff.
} 
Let $E$ be the standard conditional expectation from $C_{r}^{*}(G)$ to $C_{0}\left(G^{(0)}\right)$ [10, II.4.8], [11, 4.3]. For $f$ in $C_{c}(G)$ recall that $E(f)$ coincides with the restriction of $f$ to $G^{(0)}$, so we may write $(4.4 .2)$ as

$$
\|E(f)\| \leq\|\pi(f)\|, \quad \forall f \in C_{c}(G) .
$$

Letting $B$ be the range of $\pi$, we claim that there exists a bounded linear map $F$ from $B$ to $C_{0}\left(G^{(0)}\right)$ such that the diagram

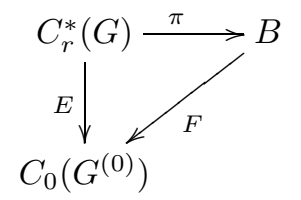

commutes. We first define $F$ on the dense ${ }^{*}$-subalgebra $\pi\left(C_{c}(G)\right) \subseteq B$ by

$$
F(\pi(f))=E(f), \quad \forall f \in C_{c}(G) .
$$

By (4.4.3) this is well defined and bounded, and hence may be continuously extended to the whole of $B$. The extension will then clearly satisfy the required conditions.

Let $a \in C_{r}^{*}(G)$ be such that $\pi(a)=0$. Then

$$
0=F\left(\pi\left(a^{*}\right) \pi(a)\right)=F\left(\pi\left(a^{*} a\right)\right)=E\left(a^{*} a\right) .
$$

Since $E$ is faithful [11, 4.3ii], we deduce that $a=0$, hence concluding the proof of (a).

We now turn to proving (b). Consider a representation $\pi$ of $C_{r}^{*}(G)$ whose kernel coincides with $J$. Such a representation may be obtained by faithfully embedding $C_{r}^{*}(G) / J$ as an algebra of operators on a Hilbert space.

Arguing by contradiction, if the intersection of $J$ with $C_{0}\left(G^{(0)}\right)$ is zero, then the restriction $\left.\pi\right|_{C_{0}\left(G^{(0)}\right)}$ is faithful and hence $\pi$ itself is faithful by (a), from which one would deduce that $J$ is zero.

\section{REFERENCES}

[1] C. Anantharaman-Delaroche and J. Renault, Amenable groupoids, Monographie de l'Enseignement Mathématique, 36, Genève, 2000. MR1799683 (2001m:22005)

[2] A. Connes, A survey of foliations and operator algebras, Operator algebras and applications, Part I (Kingston, Ont., 1980), Proc. Sympos. Pure Math., 38, Amer. Math. Soc., 1982, 521-628. MR679730 (84m:58140)

[3] J. Cuntz and W. Krieger, A class of $C^{*}$-algebras and topological Markov chains, Inventiones Math., 56 (1980), 251-268. MR.561974 (82f:46073a)

[4] R. Exel, Inverse semigroups and combinatorial C*-algebras, Bull. Braz. Math. Soc. (N.S.), 39 (2008), 191-313. MR2419901 (2009b:46115)

[5] R. Exel and M. Laca, Cuntz-Krieger algebras for infinite matrices, J. Reine Angew. Math., 512 (1999), 119-172. MR 1703078(2000i:46064)

[6] R. Exel, M. Laca and J. Quigg, Partial dynamical systems and $C^{*}$-algebras generated by partial isometries, J. Operator Theory, 47 (2002), 169-186. MR.1905819 (2003f:46108)

[7] A. Kumjian, On C*-diagonals, Canad. J. Math., 38 (1986), no. 4, 969-1008. MR854149 (88a:46060)

[8] A. Kumjian, D. Pask, and I. Raeburn, Cuntz-Krieger algebras of directed graphs, Pacific J. Math., 184 (1998), 161-174. MR1626528 (99i:46049)

[9] A. L. T. Paterson, Groupoids, inverse semigroups, and their operator algebras, Birkhäuser, 1999. MR:1724106 (2001a:22003) 
[10] J. Renault, A groupoid approach to $C^{*}$-algebras, Lecture Notes in Mathematics, vol. 793, Springer, 1980. MR584266 (82h:46075)

[11] J. Renault, Cartan subalgebras in $C^{*}$-algebras, Irish Math. Soc. Bull., 61 (2008), 29-63. MR2460017 (2009k:46135)

[12] J. Renault, The ideal structure of groupoid crossed product $C^{*}$-algebras [with an appendix by G. Skandalis], J. Operator Theory, 25 (1991), 3-36. MR.1191252 (94g:46074)

Departamento de Matemática, Universidade Federal de Santa Catarina, 88040-900, FlorianóPOLIS, BRAZIL

E-mail address: r@exel.com.br 